\title{
Numerical Analysis of Combustion Oscillation Stabilization by Helmholtz Oscillation Control*
}

\author{
Kazunori SHIODA**, Susumu YAMANAKA**, Fukuo MAEDA**, Kazunori IWABUCHI**, \\ Toshiaki TSUCHIYA ${ }^{* * * *}$ and Masanori OKAMOTO ${ }^{* * * *}$
}

\begin{abstract}
Combustion oscillation stabilization by Helmholtz resonator has been investigated for gas turbine combustor, and mechanism of oscillation control has been discussed in terms of analysis model of Helmholtz oscillation, excited between combustor and fuel injector. Amplitude of oscillation pressure is stabilized corresponding to resonator parameters, such as throat diameter and length, and calculated results on combustion oscillation stabilization are compared with experimental results by atmospheric combustion apparatus. In case Helmholtz resonator equipped to combustor is in resonance to combustor chamber, it is shown that amplitude of oscillation pressure can be minimized. However, effectiveness of the resonator may be lessened with decrease of throat diameter or increase of throat length, while throat diameter and length are both tuning parameters of the resonator. Throat resistance, which is determined by both of throat diameter and length, is actually one of the major parameters in Helmholtz oscillation control. Resonator parameters must be optimized in terms of attenuation characteristics as well as resonance frequency.
\end{abstract}

Key Words: Gas Turbine, Combustion, Oscillating Combustion, Helmholtz Oscillation, Resonator

\section{Introduction}

It is well known that gas turbine combustor is followed by combustion oscillation driven by flame in the frequency range of $10^{1}-10^{3} \mathrm{~Hz}$. Combustion oscillation, which gives rise to abnormal vibrations corresponding to resonance frequencies of combustor, is one of the selfoscillation phenomena encountered in various combustion apparatus including gas turbine combustor. Especially in

* Received 21st April, 2004 (No. 02-1025). Japanese Original: Trans. Jpn. Soc. Mech. Eng., Vol.69, No.681, B (2003), pp.1254-1261 (Received 13th August, 2004)

** Power and Industrial Systems Research and Development Center, Toshiba Corporation Industrial and Power Systems \&Services Company, 2-4 Suehiro-cho, Tsurumi-ku, Yokohama 230-0045, Japan.

E-mail: kazunori1.shioda@toshiba.co.jp

*** Power and Industrial Systems Research and Development Center, Toshiba Corporation Industrial and Power Systems \& Services Company, 1 Toshiba-cho, Fuchu, Tokyo 1838511, Japan

**** Dispersed Power Resources Technology Group R\&D Center, Engineering R\&D Division, Tokyo Electric Power Company, 4-1 Egasaki-cho, Tsurumi-ku, Yokohama 2308510, Japan combined cycle or coal gasification power generation, in which low NOx combustion or fuel variation is requested in order to minimize the influence to circumstances, occurrence of combustion oscillation coming from some combustion instabilities is observed. Recently, combustion oscillation stabilization is one of the most important problems in research and development of gas turbine combus$\operatorname{tor}^{(1)-(4)}$.

Research on combustion oscillation originated by Rayleigh $^{(5)}$ is the old theme, and many studies have been reported so $\mathrm{far}^{(6)}$. However, mechanism and specified characteristics of combustion oscillation are not yet clarified sufficiently, since combustion oscillation as selfoscillation is complicatedly related to shape of combustion chamber and surrounding systems of combustor. And it should be noticed that various patterns of combustion oscillation exist, such as intrinsic oscillation of flame due to microscopic instabilities and fluctuations of reaction ${ }^{(7)}$, acoustic oscillation in resonance to combustion chamber ${ }^{(8),(9)}$, and Helmholtz oscillation between combustor and surrounding systems of combustor ${ }^{(10)}$. Intrinsic oscillation of flame, which occurs usually in chemical reactions, is considered to be the oscillation source of acoustic and Helmholtz oscillations. 
In this work, the effectiveness of Helmholtz resonator on combustion oscillation stabilization has been evaluated in Helmholtz oscillations ${ }^{(11),(12)}$ that might induce low frequency oscillations in resonance to combustion chamber. Information on application of the resonator to combustor will be obtained by numerical analyses and atmospheric experiments.

\section{List of Symbols}

$A_{i} \quad$ : Cross section of elemnt $i\left[\mathrm{~m}^{2}\right]$

$a_{i}:$ Sound velocity in element $i[\mathrm{~m} / \mathrm{s}]$

$d \quad:$ Throat diameter [m]

$F$ : Pressure amplitude of sound source $[\mathrm{Pa}]$

$f \quad$ : Forced frequency of sound source [Hz]

$f_{i} \quad$ : Helmholtz frequency of element $i[\mathrm{~Hz}]$

$L_{i} \quad$ : Length of element $i[\mathrm{~m}]$

$l_{P} \quad:$ Piston location [m]

$\bar{M}$ : Averaged molecular weight $[\mathrm{kg} / \mathrm{kmol}]$

$M_{i}$ : Fluid mass in element $i[\mathrm{~kg}]$

$M_{i j}$ : Reduced fluid mass in element $i$ and $j[\mathrm{~kg}]$

$p_{i}$ : Pressure in element $i[\mathrm{~Pa}]$

$R$ : Gas constant $[\mathrm{J} / \mathrm{mol} \cdot \mathrm{K}]$

$R_{i} \quad$ : Resistance of element $i\left[\mathrm{~m}^{-1} \mathrm{~s}^{-1}\right]$

$T_{i}$ : Temperature in element $i[\mathrm{~K}]$

$t \quad:$ Time [s]

$u_{i}$ : Flow velocity in element $i[\mathrm{~m} / \mathrm{s}]$

$V_{i}:$ Volume of element $i\left[\mathrm{~m}^{3}\right]$

$\gamma \quad$ : Ratio of specific heat [-]

$\rho_{i} \quad$ : Mass density of fluid in element $i\left[\mathrm{~kg} / \mathrm{m}^{3}\right]$

$\phi_{i} \quad$ : Mass flux in element $i[\mathrm{~kg} / \mathrm{s}]$

$\varphi_{i} \quad$ : Phase shift in element $i[\mathrm{rad}]$

$\omega$ : Forced angular frequency [rad/s]

$\omega_{i}$ : Helmholtz angular frequency of element $i$ $[\mathrm{rad} / \mathrm{s}]$

Subscripts

0 : Stationary

$C$ : Combustion chamber

$F \quad:$ Fuel injector

$R$ : Resonator

\section{Numerical Analysis}

\section{1 Helmholtz oscillation model}

In Fig. 1 each type of combustion oscillation is shown together with components of combustor equipped with resonator. Combustion oscillation has three typical patterns of oscillation, which are intrinsic oscillation, acoustic oscillation and Helmholtz oscillation respectively. As shown in Table 1, three types of oscillations occur in flame, in combustor chamber, and between combustor and fuel injector, respectively.

Intrinsic oscillation is the pressure oscillation having various frequencies due to periodic fluctuation of heat release in flame, coming from diffusive combustion of fuel and air. Acoustic oscillation is excited in combustion

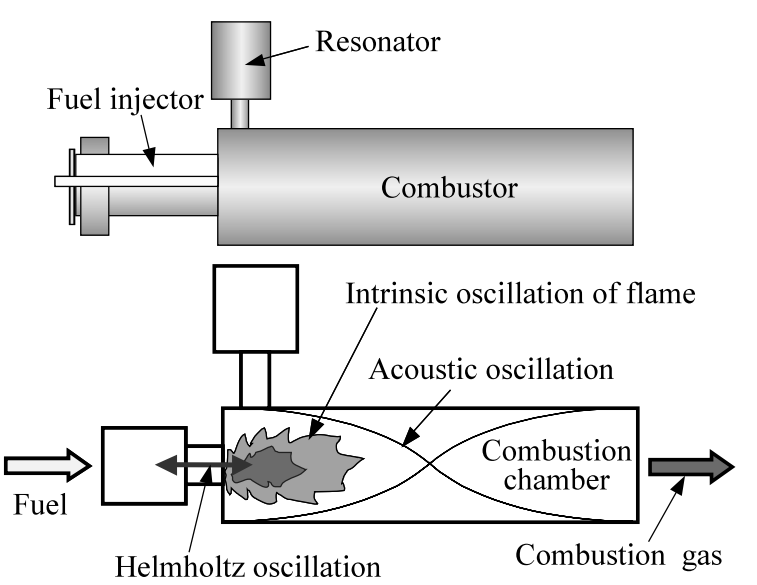

Fig. 1 Schematic view of typical oscillation around combustor

Table 1 Typical oscillation around combustor

\begin{tabular}{c|c}
\hline Oscillation type & Place of occurrence \\
\hline \hline Intrinsic oscillation & Flame \\
\hline Acoustic oscillation & Combustion chamber \\
\hline Helmholtz oscillation & $\begin{array}{c}\text { Between combustor } \\
\text { and fuel injector }\end{array}$ \\
\hline \hline
\end{tabular}

chamber by flame, and according to the shape of combustion chamber it has some prominent resonance frequencies in the range of $100 \mathrm{~Hz}-1 \mathrm{MHz}$. Combustion apparatus used here, as described in section 4.1, has orifice plate at its end assuming stationary blades of gas turbine. In this case acoustic oscillation mode shown in Fig. 1 has node of pressure amplitude at the center of combustion chamber, and loops both at entrance and exit of combustion chamber. And Helmholtz oscillation is driven by flame in a certain fluid oscillation system such between combustor and fuel injector. Therefore, combustion oscillation may be referred to acoustic and Helmholtz oscillations driven by intrinsic oscillation of flame. However, mechanism and specified characteristics of combustion oscillations are not necessarily clarified since they interfere complicatedly with each other.

Here, Helmholtz oscillation stabilization using resonator, excited between combustor and fuel injector, is investigated in terms of numerical analyses and experiments.

As shown in Fig. 2, resonator has simple structure with throat attached to cavity. Compressible fluid filled in cavity produces oscillatory flow in throat, which is so called Helmholtz oscillation. In localized constant model of resonator shown in Fig. 2, fluid in cavity, fluid in throat, and pressure drop in throat are represented by spring constant $k_{R}$, mass $m_{R}$ and resistance $R_{R}$ respectively. And resonance angular frequency is determined by these localized constants. Figure 3 shows localized constant model of relaxation system by resonator, which corresponds to coupling oscillation system composed of combustor, fuel 


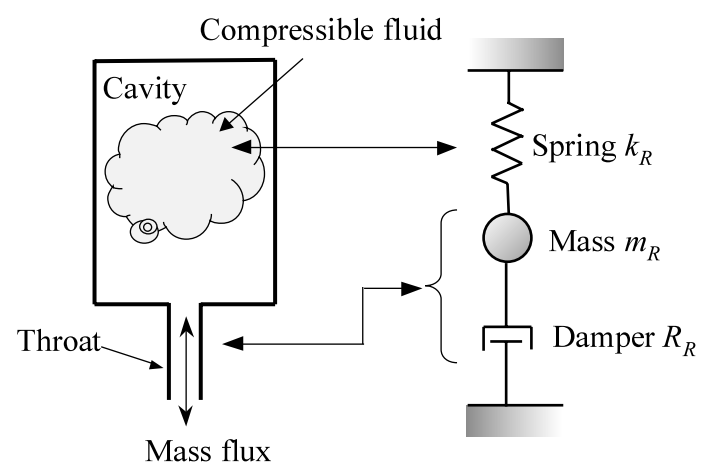

Fig. 2 Localized constant model of resonator

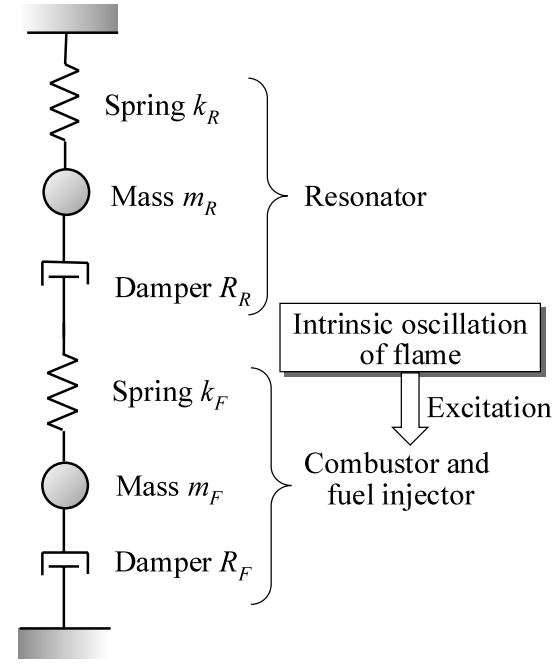

Fig. 3 Localized constant model of relaxation system by resonator

injector, and resonator as shown in Fig. 1. Here, fluid in combustion chamber and fuel injector, fluid in fuel pipe connecting between combustion chamber and fuel injector, and pressure drop in fuel pipe are represented by spring constant $k_{F}$, mass $m_{F}$, and resistance $R_{F}$ respectively. The system described here is excited by intrinsic oscillation of flame.

Figure 4 shows the schematic view of the effect of combustion oscillation stabilization by resonator. Oscillation response to forced frequency with and without resonator is depicted in Fig. 4, where ordinate is pressure amplitude of oscillation response, and abscissa is forced angular frequency. It can be assumed here that single response angular frequency $\omega_{H}$, which is called Helmholtz angular frequency and is determined by dimensions of each component, is excited between combustion chamber and fuel injector if combustor is not equipped with resonator. In case forced angular frequency $\omega$ of flame is nearly equal to Helmholtz angular frequency $\omega_{H}$, system is in resonance.

If resonator is equipped to combustor and in case its throat resistance $R_{R}$ is large, single resonance peak displayed in dotted line in Fig. 4 is expected to relax since

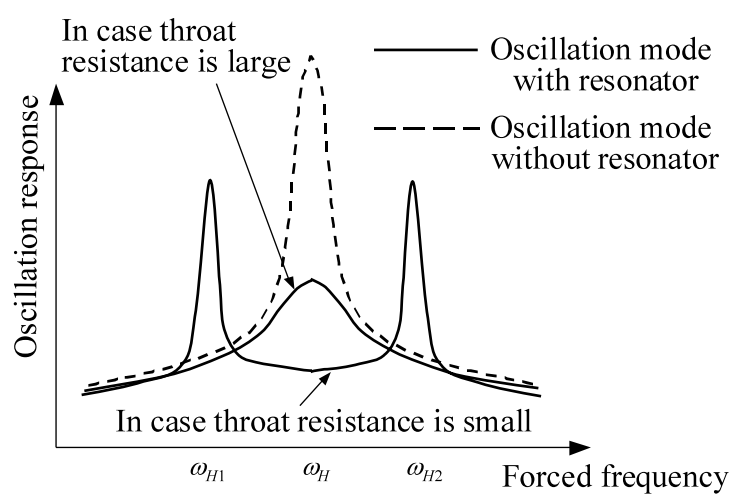

Fig. 4 Oscillation response to forced frequency

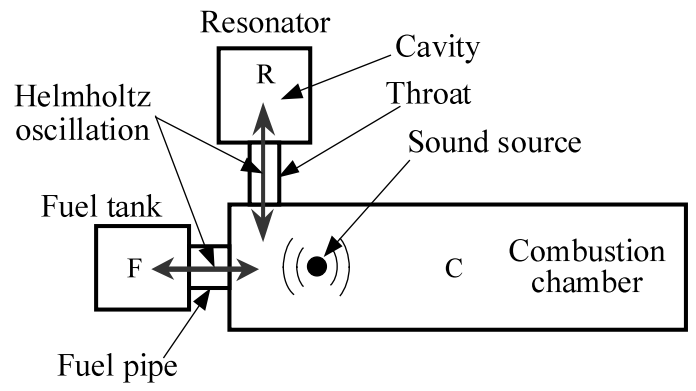

Fig. 5 Element model of Helmholtz oscillation

oscillation energy is absorbed in throat. On the other hand, two resonance peaks appear in case throat resistance $R_{R}$ is small, corresponding to resonance angular frequencies $\omega_{H 1}, \omega_{H 2}$, which represent oscillation modes of same phase and inverse phase respectively. In mode of same phase masses $m_{F}$ and $m_{R}$ shown in Fig. 3 vibrate in same direction, and in mode of inverse phase masses $m_{R}$ and $m_{R}$ vibrate in inverse direction. After all, by equipping resonator to combustor resonance angular frequency of system can be shifted from Helmholtz angular frequency $\omega_{H}$ to other resonance angular frequencies $\omega_{H 1}, \omega_{H 2}$.

\subsection{Governing equations}

Oscillation response characteristics to forced frequency of sound source, based on the Helmholtz vibration model in Fig. 3, are numerically evaluated in terms of element model in Fig. 5 with mutual interactions of combustion chamber, fuel injector, resonator and connecting pipes between them. Numerical analysis here deals with time dependent component of flow, although fuel and combustion gas make almost stationary flow in combustor. And three approximations described below, same phase, isentropic, and linear approximations respectively, hold in Helmholtz vibration model.

Firstly according to the same phase approximation, it can be assumed that since sound velocity is infinite physical quantity in a certain element such as pressure is unique and changes in same phase. As shown in Eq. (1), the equation of fluid motions in fuel pipe and throat of resonator, pressure $p_{i}$ in element $i$ and mass flux $\phi_{i}$ in each pipe be- 
tween combustion chamber and element $i$ are interacting with each others. Mass flux $\phi_{i}$ is unique regardless of location in pipe $i$, and the directions of $\phi_{i}$ from chamber are assumed to be positive here. In Eq. (1) $A_{i}$ stands for cross section $\left[\mathrm{m}^{2}\right], L_{i}$ for length [m], $p_{i}$ for pressure [Pa], $R_{i}$ for resistance $\left[\mathrm{m}^{-1} \mathrm{~s}^{-1}\right], u_{i}$ for flow velocity $[\mathrm{m} / \mathrm{s}]$, and $\rho_{i}$ for mass density in element $i\left[\mathrm{~kg} / \mathrm{m}^{3}\right]$, where subscript $C, F$ and $R$ denote combustion chamber, fuel injector and resonator respectively.

$$
L_{i} \frac{d \phi_{i}}{d t}+A_{i} R_{i} \phi_{i}+A_{i}\left(p_{i}-p_{C}\right)=0 \quad(i=F, R)
$$

where, $\phi_{i}=\rho_{i} u_{i} A_{i}$

Secondly according to the isentropic approximation, Eq. (2) is obtained if exhaust process of fluid from element through pipe is considered as isentropic process, that is $p \propto \rho^{\gamma}$, in which $\gamma$ is specific heat ratio.

$$
\frac{d p_{i}}{\gamma p_{i}}=\frac{d \rho_{i}}{\rho_{i}} \quad(i=C, F, R)
$$

Thirdly according to the linear approximation, Eq. (3) is obtained under the condition that fluid mass $M_{i}$ in element $i$ is nearly constant and time dependent components of pressure $p_{i}$ are sufficiently small compared with time independent component of them, that is $\bar{p}_{i} \gg p_{i}^{\prime}$, where $\bar{p}_{i}$ and $p_{i}^{\prime}$ represents time dependent and time dependent components of pressure $[\mathrm{Pa}]$.

$$
\left\{\begin{array}{l}
M_{i} \approx \text { const. } \\
p_{i}=\bar{p}_{i}+p_{i}^{\prime} \approx p_{0}
\end{array}(i=C, F, R)\right.
$$

Equation (4), which displays the relation between pressure $p_{i}$ and mass flux in pipe $\phi_{i}$, is obtained by Eq. (2) using Eq. (3). As for the pressure $p_{C}$ in combustion chamber, the first term in right side of the first equation of Eq. (4) contains sound source $p_{f}=F \cdot \sin \omega t$, where F represents pressure amplitude $[\mathrm{Pa}], \omega$ for forced angular frequency $[\mathrm{rad} / \mathrm{s}]$. And the second term of the first equation, and the first term of the second equation of Eq. (4) denote pressure change in time due to mass exhaust to fuel injector and resonator.

Equation (5) as vibration equation is obtained by substituting Eq. (4) into Eq. (1), in which right side denotes sound source.

$$
\frac{1}{\gamma p_{0}} \frac{d p_{i}}{d t}= \begin{cases}\frac{F \omega}{\gamma p_{0}} \cos \omega t-\frac{1}{M_{i}} \sum_{j \neq i} \phi_{j} & (i=C) \\ \frac{\phi_{i}}{M_{i}} & (i=F, R)\end{cases}
$$

where, $\omega=2 \pi f$

$$
\left\{\begin{array}{l}
\frac{d^{2} \phi_{F}}{d t^{2}}+\frac{A_{F} R_{F}}{L_{F}} \frac{d \phi_{F}}{d t}+\omega_{C F}^{2} \phi_{F}+\omega_{F}^{2} \phi_{R}=\frac{A_{F} F \omega}{L_{F}} \cdot \cos \omega t \\
\frac{d^{2} \phi_{R}}{d t^{2}}+\frac{A_{R} R_{R}}{L_{R}} \frac{d \phi_{R i}}{d t}+\omega_{C R}^{2} \phi_{R}+\omega_{R}^{2} \phi_{F}=\frac{A_{R} F \omega}{L_{R}} \cdot \cos \omega t
\end{array}\right.
$$

Helmholtz angular frequencies $\omega_{F}$ and $\omega_{R}$, introduced in Eq. (5), are given in Eq. (6) in exhaust from combustion chamber to atmosphere through fuel pipe and throat of resonator respectively. And Helmholtz angular frequencies $\omega_{C F}$ and $\omega_{C R}$ between combustion chamber and fuel injector, and resonator respectively are also given in Eq. (6). According to temperature $T_{i}$ and volume $V_{i}$ of each element $i$, sound velocity $a_{i}$ and equivalent sound velocity $a_{C i}$ in each connecting pipes, and reduced fluid mass $M_{C i}$ of combustion chamber and each element $i$ are defined in Eqs. (7) and (8).

$$
\left\{\begin{array}{l}
\omega_{i}=\sqrt{\frac{A_{i} \gamma p_{0}}{L_{i} M_{C}}}=a_{C} \sqrt{\frac{A_{i}}{L_{i} V_{C}}} \\
\omega_{C i}=\sqrt{\frac{A_{i} \gamma p_{0}}{L_{i} M_{C i}}}=a_{C i} \sqrt{\frac{A_{i}}{L_{i}}\left(\frac{1}{V_{C}}+\frac{1}{V_{i}}\right)}
\end{array}\right.
$$

where, $\omega_{i}=2 \pi f_{i}, \omega_{i j}=2 \pi f_{i j}$

$$
\begin{aligned}
& a_{i}=\sqrt{\gamma \frac{p_{0}}{\rho_{i}}}=\sqrt{\gamma \frac{R T_{i}}{\bar{M}}} \\
& a_{C i}=\sqrt{\frac{\gamma \frac{R}{\bar{M}}\left(\frac{T_{C}}{V_{C}}+\frac{T_{i}}{V_{i}}\right)}{\frac{1}{V_{C}}+\frac{1}{V_{i}}}} \\
& M_{C i}=\left(\frac{1}{M_{C}}+\frac{1}{M_{i}}\right)^{-1} \quad(i=F, R)
\end{aligned}
$$

Vibration equation shown in Eq. (5), inhomogeneous differential equation of second order with constant coefficients, gives general solution as linear combination of natural and response vibration terms. Since amplitude of natural vibration decreases exponentially as time goes to infinity $t \rightarrow \infty$, Eq. (9) shows only response vibration term which survives as long as sound source exists. Coefficients $C_{k}(k=1-4)$ are obtained by solving Eq. (10), which is given by substituting Eq. (9) into Eq. (5).

$$
\left\{\begin{array}{l}
\phi_{F}=C_{1} \cdot \cos \omega t+C_{2} \cdot \sin \omega t=\sqrt{C_{1}^{2}+C_{2}^{2}} \cdot \cos \left(\omega t-\varphi_{F}\right) \\
\phi_{R}=C_{3} \cdot \cos \omega t+C_{4} \cdot \sin \omega t=\sqrt{C_{3}^{2}+C_{4}^{2}} \cdot \cos \left(\omega t-\varphi_{R}\right)
\end{array}\right.
$$

$$
\begin{aligned}
& {\left[\begin{array}{c}
C_{1} \\
C_{2} \\
C_{3} \\
C_{4}
\end{array}\right]=\left[\begin{array}{cccc}
\omega_{C F}^{2}-\omega^{2} & \frac{A_{F} R_{F} \omega}{L_{F}} & \omega_{F}^{2} & 0 \\
-\frac{A_{F} R_{F} \omega}{L_{F}} & \omega_{C F}^{2}-\omega^{2} & 0 & \omega_{F}^{2} \\
\omega_{R}^{2} & 0 & \omega_{C R}^{2}-\omega^{2} & \frac{A_{R} R_{R} \omega}{L_{R}} \\
0 & \omega_{R}^{2} & -\frac{A_{R} R_{R} \omega}{L_{R}} & \omega_{C R}^{2}-\omega^{2}
\end{array}\right]^{-1} } \\
& \cdot\left[\begin{array}{c}
\frac{A_{F} F \omega}{L_{F}} \\
0 \\
\frac{A_{R} F \omega}{L_{R}} \\
0
\end{array}\right]
\end{aligned}
$$

Equations (11) and (12) denote time dependent component of pressure in each element in Eq. (4) and phase delay of mass flux in each pipe to sound source $p_{f}=F \cdot \sin \omega t$ in Eq. (9). 


$$
\begin{aligned}
& \left\{\begin{aligned}
p_{C}= & \frac{\gamma p_{0}}{M_{C} \omega} \sum_{j=1}^{2} C_{2 j} \cdot \cos \omega t+\left(F-\frac{\gamma p_{0}}{M_{C} \omega} \sum_{j=1}^{2} C_{2 j-1}\right) \\
& \cdot \sin \omega t \\
p_{F}= & \frac{\gamma p_{0}}{M_{F} \omega}\left(-C_{2} \cdot \cos \omega t+C_{1} \cdot \sin \omega t\right) \\
p_{R}= & \frac{\gamma p_{0}}{M_{R} \omega}\left(-C_{4} \cdot \cos \omega t+C_{3} \cdot \sin \omega t\right)
\end{aligned}\right. \\
& \left\{\begin{array}{l}
\varphi_{F}=\tan ^{-1} \frac{C_{2}}{C_{1}} \\
\varphi_{R}=\tan ^{-1} \frac{C_{4}}{C_{3}}
\end{array}\right.
\end{aligned}
$$

\section{Calculated Results}

\section{1 Analysis conditions}

Combustion oscillation stabilization by Helmholtz resonator is investigated here by means of atmospheric experiments. Figure 6 shows the schematic view of atmospheric experimental apparatus used here, which has orifice plate at its end assuming stationary blades of gas turbine. Combustion chamber has $1.5 \mathrm{~m}$ of length and $0.224 \mathrm{~m}$ of inner diameter, and cross sectional ratio of orifice plate is 0.227 . Using natural gas as fuel and by controlling temperature of entrance air up to $350^{\circ} \mathrm{C}$, maximum combustion temperature of $1600^{\circ} \mathrm{C}$ is obtained.

Pressure oscillation in combustion chamber was measured by piezo dielectric sensors with frequency analyzing

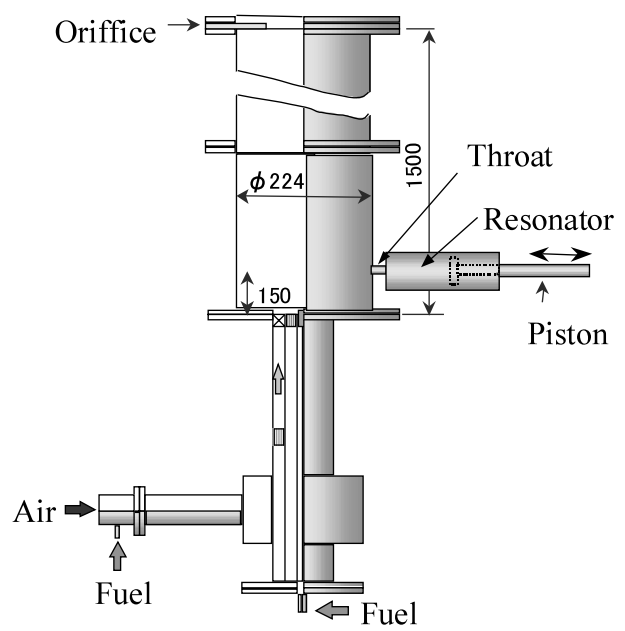

Fig. 6 Schematic view of experimental apparatus apparatus via amplifier. Consequently two prominent resonance peaks at $70 \mathrm{~Hz}$ and $270 \mathrm{~Hz}$ were confirmed in practice, and pressure oscillation mode of $70 \mathrm{~Hz}$ can be specified as Helmholtz oscillation between combustion chamber and fuel injector, since pressures in all locations of combustion chamber are in same phase. On the other hand, pressure oscillation mode of $270 \mathrm{~Hz}$ is specified as acoustic oscillation, since pressures in upstream and downstream of combustion chamber are in inverse phase as illustrated in Fig. 1.

By adjusting Helmholtz angular frequency $\omega_{C R}$ to the neighborhood of $\omega_{C F}$, system is no longer in resonance and pressure amplitude in combustion chamber is expected to relax to some degree. Table 2 shows properties of combustion gas and Table 3 shows dimensions of elements. Dimensions of fuel injector in Table 3 were assumed so that Helmholtz frequency $\omega_{C R}$ between combustion chamber and fuel injector be $70 \mathrm{~Hz}$, as confirmed experimentally. Table 4 shows analysis conditions on temperature, pressure, resistance and sound source.

\section{2 Frequency response characteristics}

Figure 7 shows calculated results of frequency response characteristics to forced frequency of sound source, where ordinate is pressure amplitude $p_{C}$ in combustion chamber, and abscissa is forced angular frequency $\omega$. Although sound source in practice has white spectra in angular frequency, it is assumed in numerical analysis to have the single forced angular frequency $\omega$. And by sweeping forced angular frequency $\omega$ one can obtain fre-

Table 2 Properties of combustion gas

\begin{tabular}{c|c}
\hline \hline Molecular weight $\bar{M}$ & $28.34 \mathrm{~kg} / \mathrm{kmol}$ \\
\hline Specific heat ratio $\gamma$ & 1.278 \\
\hline Gas constant $R / \bar{M}$ & $293.4 \mathrm{~J} / \mathrm{kgK}$ \\
\hline Viscosity $\mu$ & $6.290 \times 10^{-5} \mathrm{~Pa}^{*} \mathrm{~s}$ \\
\hline \hline
\end{tabular}

Table 3 Dimensions of elements

\begin{tabular}{c|c|c|c}
\hline \multicolumn{2}{|c|}{} & Diameter & Length \\
\hline \multicolumn{2}{c|}{ Combustor } & $224 \mathrm{~mm}$ & $1500 \mathrm{~mm}$ \\
\hline \multirow{2}{*}{ Resonator } & Cavity & $151 \mathrm{~mm}$ & Variable \\
\cline { 2 - 4 } & Throat & $25 \mathrm{~mm}$ & $55 \mathrm{~mm}$ \\
\hline \multirow{2}{*}{ Fuel injector } & Tank & $200 \mathrm{~mm}$ & $500 \mathrm{~mm}$ \\
\cline { 2 - 4 } & Pipe & $51 \mathrm{~mm}$ & $200 \mathrm{~mm}$ \\
\hline
\end{tabular}

\begin{tabular}{|c|c|c|c|c|c|}
\hline & & Temperature & Pressure & Resistance & Sound source \\
\hline \multicolumn{2}{|c|}{ Combustor } & $T_{C}=1800 \mathrm{~K}$ & \multirow{5}{*}{$1.013 \times 10^{5} \mathrm{~Pa}$} & No resistance added & $\begin{array}{l}\text { Amplitude } \\
F=100 . \mathrm{Pa}\end{array}$ \\
\hline \multirow{2}{*}{ Resonator } & Cavity & \multirow{2}{*}{$T_{R}=460 \mathrm{~K}$} & & \multirow{2}{*}{$\begin{array}{c}R_{R}=1 . \times 10^{4} \mathrm{~m}^{-1} \mathrm{~s}^{-1} \\
\sim 1 . \times 10^{5} \mathrm{~m}^{-1} \mathrm{~s}^{-1}\end{array}$} & \multirow{4}{*}{$\begin{array}{c}\text { No sound } \\
\text { source added }\end{array}$} \\
\hline & Throat & & & & \\
\hline \multirow{2}{*}{$\begin{array}{l}\text { Fuel } \\
\text { injector }\end{array}$} & Tank & \multirow{2}{*}{$T_{F}=300 \mathrm{~K}$} & & \multirow{2}{*}{$R_{F}=5 . \times 10^{3} \mathrm{~m}^{-1} \mathrm{~s}^{-1}$} & \\
\hline & Pipe & & & & \\
\hline
\end{tabular}

Table 4 Standard analysis conditions 


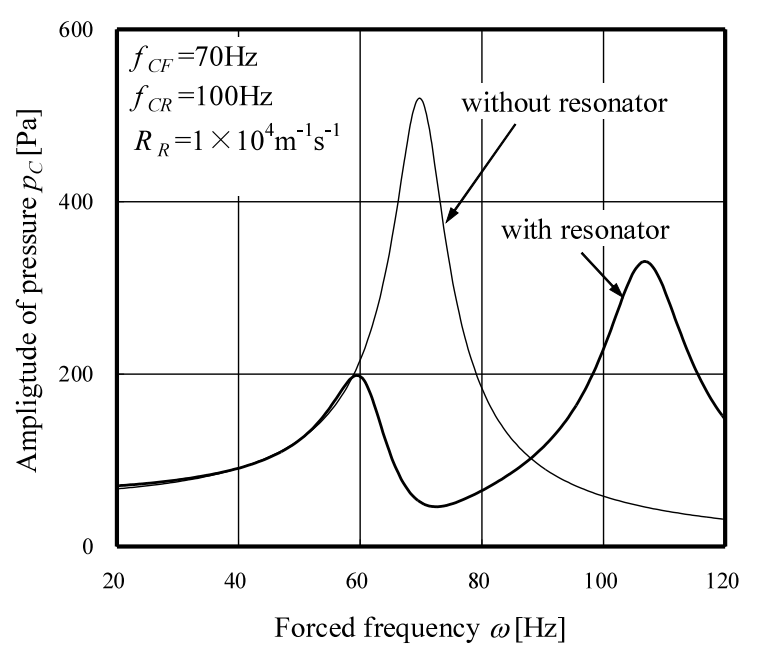

Fig. 7 Response characteristics to forced oscillation in case throat resistance is $R_{R}=1 \times 10^{4} \mathrm{~m}^{-1} \mathrm{~s}^{-1}$

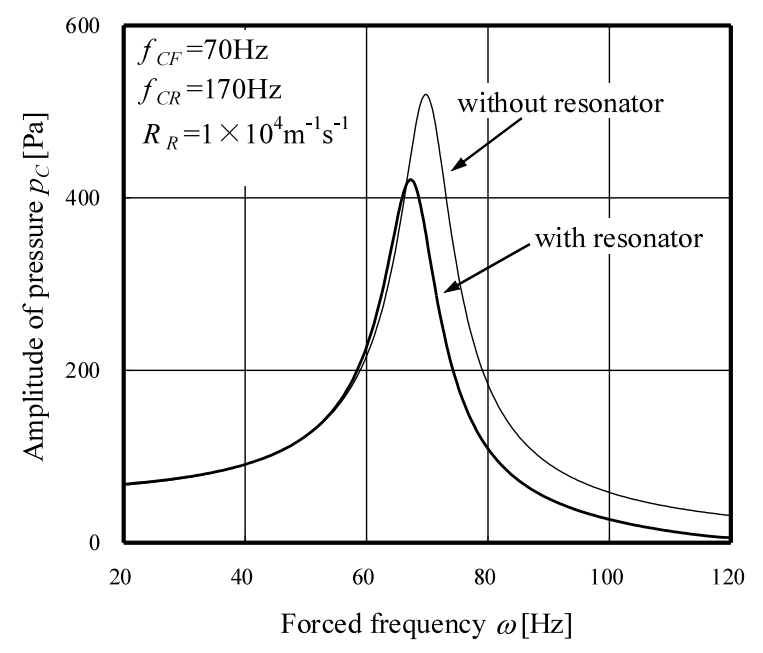

Fig. 8 Response characteristics to forced oscillation in case throat resistance is $R_{R}=1 \times 10^{5} \mathrm{~m}^{-1} \mathrm{~s}^{-1}$

quency response characteristics. In case combustor is not equipped with resonator Helmholtz oscillation appears to have its resonance peak at $70 \mathrm{~Hz}$ as shown by fine line, and it responds to sound source of $70 \mathrm{~Hz}$. For example with resonator of Helmholtz frequency $f_{C R}=100 \mathrm{~Hz}$ and throat resistance $R_{R}=1 \times 10^{4} \mathrm{~m}^{-1} \mathrm{~s}^{-1}$, two resonance peaks appeared as shown by solid line. In this case system does not respond to sound source since system is no longer in resonance to sound source. Figure 8 shows frequency response characteristics with resonator of Helmholtz frequency $f_{C R}=170 \mathrm{~Hz}$. Contrary to Fig. 7, resonance peak is not so relaxed since frequency response characteristics are not affected by resonator.

Large pressure amplitude excited in combustion chamber, in case it is in resonance to sound source, can be relaxed effectively by resonator which is in resonance to combustion chamber. Throat resistance $R_{R}$ is one of the major factors and important parameters here for oscillation control. Figure 9 shows frequency response char-

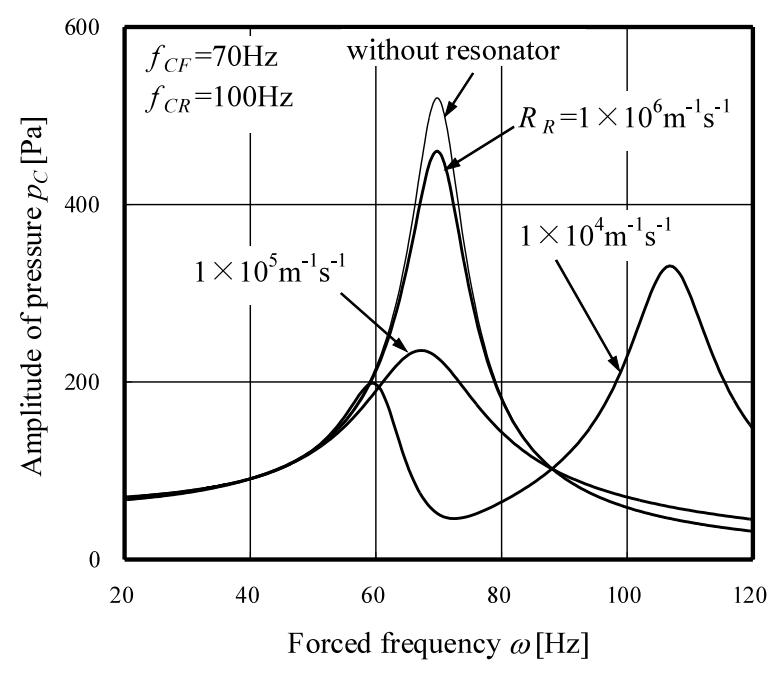

Fig. 9 Dependence to throat resistance in response characteristics

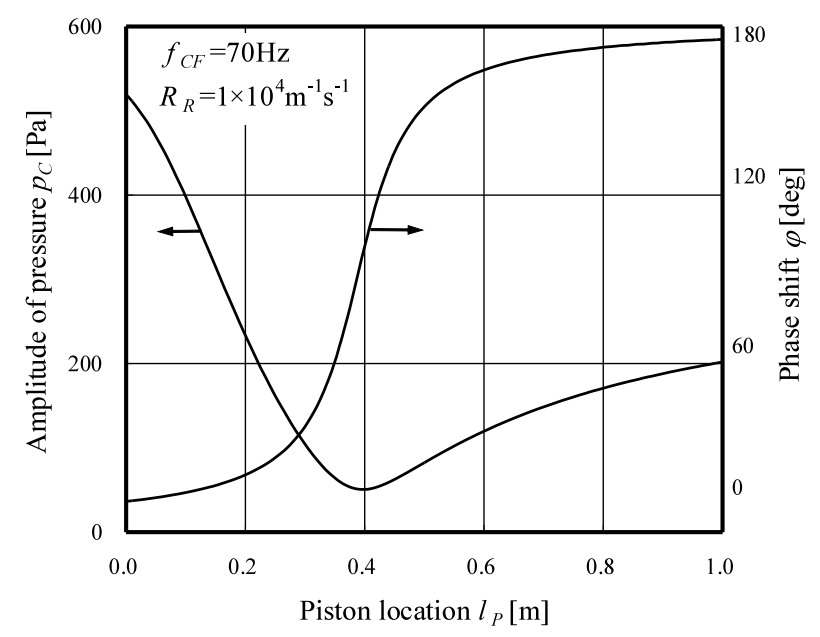

Fig. 10 Tuning characteristics

acteristics with resonator of Helmholtz frequency $f_{C R}=$ $100 \mathrm{~Hz}$. Since Helmholtz oscillation involves periodic flow change, if throat resistance $R_{R}$ is too large single resonance peak is not relaxed. On the other hand, if throat resistance $R_{R}$ is too small single resonance peak splits into two resonance peaks in other frequency zone.

\subsection{Effect of Oscillation Control}

Oscillation control by resonator is performed in practice by changing the length of cavity of resonator by piston drive. Figure 10 shows change of amplitude of oscillation pressure, excited by sound source of $f=70 \mathrm{~Hz}$, by resonator of throat resistance $R_{R}=1 \times 10^{4} \mathrm{~m}^{-1} \mathrm{~s}^{-1}$, where ordinates are amplitude of oscillation pressure $p_{C}$ of frequency $f=70 \mathrm{~Hz}$ in combustion chamber, and phase shift of resonator from combustion chamber, abscissa is piston location $l_{P}$. Here, piston location of $l_{P}=0$ means the condition of volume of cavity is $V_{R}=0$. As piston location $l_{P}$ is increased, amplitude of pressure $p_{C}$ is minimized in the course of operation of resonator, that is under the on- 


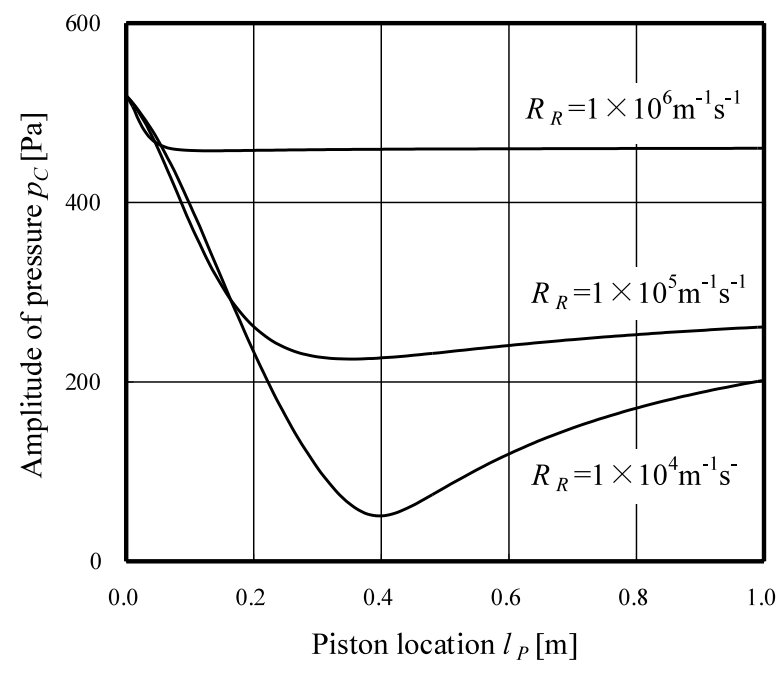

Fig. 11 Dependence to throat resistance in tuning characteristics

tuning condition. And as piston location $l_{P}$ is increased further, amplitude of pressure $p_{C}$ turns to increase again. Phase shift $\varphi$ is small in case volume of cavity is small, that is, pressure $p_{C}$ in combustion chamber oscillates almost in same phase with pressure in resonator $p_{R}$. On the other hand, phase shift $\varphi$ is near $180^{\circ}$ in case volume of cavity is large, that is, pressures $p_{C}$ and $p_{R}$ oscillate with inverse phase. Under the on-tuning condition, which shows minimum of pressure $p_{C}$, phase shift $\varphi$ is not $0^{\circ}$ or $180^{\circ}$ but around $90^{\circ}$. Figure 11 shows change of amplitude of oscillation pressure $p_{C}$, where throat resistance $R_{R}$ is parameter. As described in Fig. 9, in case throat resistance $R_{R}$ is too large Helmholtz oscillation control is not effective even if resonator in under the on-tuning condition.

\section{4 Influences of throat dimensions of resonator}

Effect of Helmholtz oscillation control can be still improved by optimum design of resonator. Here, regarding to influences on tuning characteristics due to throat dimensions of resonator, calculated results are compared with experimental results. Figure 12 shows relationship between amplitude of pressure $p_{C}$ and piston location $l_{P}$, where throat diameter $d$ is parameter. Ordinate, amplitude of pressure $p_{C}$ in combustion chamber, is non dimensioned by the value without resonator, and throat diameters are taken three values of $d=25 \mathrm{~mm}$ (standard), $20 \mathrm{~mm}, 14 \mathrm{~mm}$. Experimental results, indicated by $\bigcirc$ in Fig. 12, show good agreement with calculated results. And in Fig. 13 calculated results are shown, where throat length $L_{R}$ is parameter. Here, resistance of standard throat, corresponding to diameter $d=25 \mathrm{~mm}$ and length $L_{R}=55 \mathrm{~mm}$, is taken to be $R_{R}=1 \times 10^{5} \mathrm{~m}^{-1} \mathrm{~s}^{-1}$. And resistance of non standard throat is assumed to be inversely proportional to 4-th power of throat diameter $d$, and is proportional to throat length $L_{R}$. Equation (13) indicates that multiplication of throat resistance $R_{R}$ and mass flux $\phi_{i}$ of throat is propor-

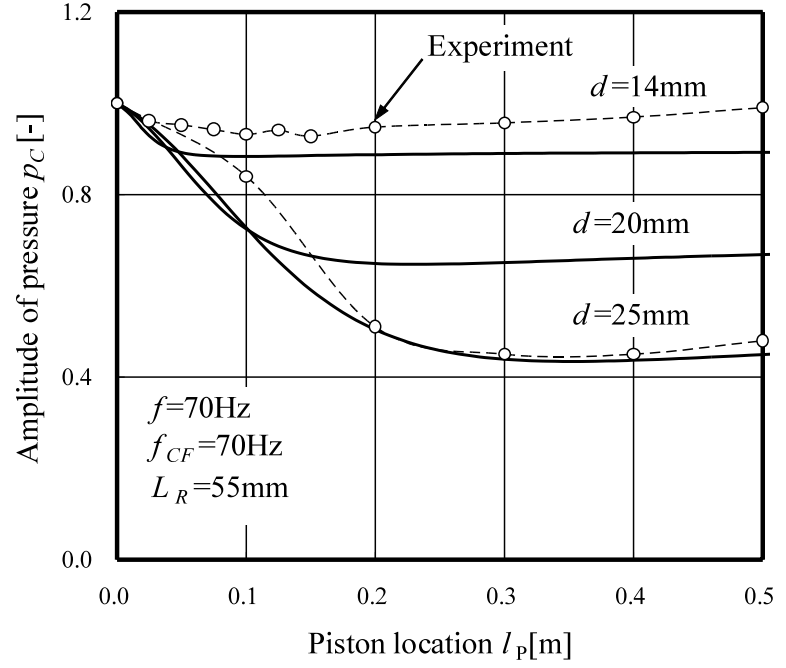

Fig. 12 Dependence to throat diameter in tuning characteristics

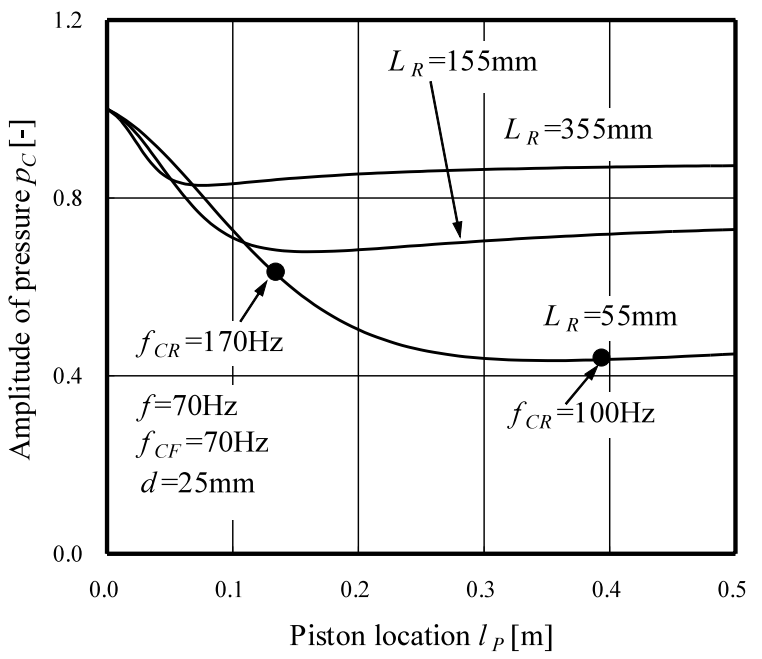

Fig. 13 Dependence to throat length in tuning characteristics

tional to pressure drop. As for coefficient of frictional loss $\zeta$ formula of laminar flow is adopted here, where $R e$ denotes Reynolds number. Hence Eq. (14) is introduced as dependence of throat resistance $R_{R}$ to throat dimensions.

$$
R_{R} \phi_{R}=\varsigma \frac{L_{R}}{d} \frac{\rho}{2} u_{R}^{2} \propto \frac{L_{R}}{d^{4}}
$$

where, $\phi_{R}=\frac{\pi}{4} d^{2} \rho u_{R}, \varsigma=\frac{64}{R e}$

$$
R_{R} \propto \frac{L_{R}}{d^{4}}
$$

Since Helmholtz angular frequency $\omega_{C R}$ of resonator has already been introduced in Eq. (6), Eq. (15) is obtained assuming that volume of cavity is sufficiently small compared with that of combustion chamber $V_{C} \gg V_{R}$.

$$
\omega_{C R} \propto \sqrt{\frac{A_{R}}{L_{R} V_{R}}} \propto \sqrt{\frac{d^{2}}{L_{R} l_{P}}}
$$

As mentioned above, it was confirmed that pressure $p_{C}$ in combustion chamber decreases by adjusting resonance frequency $f_{C R}$ to near Helmholtz frequency $f_{C F}$. 
And resonance frequencies $f_{C R}=100 \mathrm{~Hz}$ and $170 \mathrm{~Hz}$ are indicated in standard throat in Fig. 13 by $\mathcal{O}$. In order to make on-tuning condition in practice, $f_{C R}$ should be set near $100 \mathrm{~Hz}$, larger than $f_{C F}=70 \mathrm{~Hz}$. According to Eq. (15) resonator with small throat diameter $d$ or large throat length $L_{R}$ is not practical, since small piston location $l_{P}$ is needed to make on-tuning condition, and in such case throat resistance $R_{R}$ tends to be larger and oscillation control by resonator is no longer effective.

\section{Conclusions}

In this work, as for Helmholtz oscillation excited between combustion chamber and fuel injector, its mechanism was investigated by numerical analyses and atmospheric experiments. Validity of analysis model was confirmed by comparison between calculated and experimental results. Although resonator has been known as one of means of combustion oscillation stabilization so far, specification of applying resonator is not necessarily given. Analysis model proposed in this work is expected to provide effective informations on parameter design of resonator.

\section{References}

( 1 ) Richards, G.A. and Janus, M.C., Characterization of Oscillations during Premix Gas Turbine Combustion, Trans. ASME, Vol.120 (1998), pp.294-302.

( 2 ) Hoffmann, S., Lenze, B. and Eickhoff, H., Results of Experiments and Models for Predicting Stability Limits of Turbulent Swirling Flames, Trans. ASME,
Vol.120 (1998), pp.311-316.

( 3 ) Seume, J.R., Vortmeyer, N., Krause, W., Hermann, J., Hantschik, C.-C., Zangl, P., Gleis, S., Vortmeyer, D. and Orthmann, A., Trans. ASME, J. Eng. Gas Turbines and Power, Vol.120 (1998), pp.721-726.

( 4 ) Gupta, S.B., Sheshadri, T.S. and Jain, V.K., Acoustic Energy Measurements in A Coal-Burning Rijke-Tube Combustor, Int. J. Energy Research, Vol.17 (1993), pp.217-220.

( 5 ) Rayleigh, L., Theory of Sound, 2, (1945), Dover Press.

( 6 ) Putnum, A.A., Combustion-Driven Oscillations in Industry, (1971), American Elsevier Publishing Company Inc.

( 7 ) Akiyama, I., Thermoacoustic Analysis of CombustionDriven Oscillation, Trans. Jpn. Soc. Mech. Eng., (in Japanese), Vol.61, No.588, B (1995), pp.3082-3091.

( 8 ) Bloxsidge, G.J., Dowling, A.P. and Langhorne, P.J., Reheat Buzz: An Acoustically Coupled Combustion Instability Part 2 Theory, J. Fluid Mech., Vol.193 (1988), pp.445-473.

( 9 ) Keller, J.J., Thermoacoustic Oscillations in Combustion Chambers of Gas Turbines, AIAA Journal, Vol.33, No.12 (1995), pp.2280-2287.

(10) Gulati, A. and Mani, R.J., Active Control of Unsteady Combustion-Induced Oscillations, J. Propulsion and Power, Vol.8, No.5 (1992), pp.1109-1115.

(11) Ogoshi, H., Koizumi, M. and Kishimoto, K., Trans. Jpn. Soc. Mech. Eng., (in Japanese), Vol.48, No.426, B (1982), pp.373-380.

(12) Ishi, T., Kishimoto, K. and Nagata, K., Trans. Jpn. Soc. Mech. Eng., (in Japanese), Vol.50, No.449, B (1984), pp.151-158. 